\title{
Improvement of Periorbital Wrinkles Treated with an Invasive Non-Insulated Microneedle Pulsed Electric Signal Device
}

\author{
Suhyun Cho \\ Yoon Jin Choi \\ Jin-Soo Kang
}

Kangskin Dermatology Clinic, Seoul, Korea
Received January 7, 2016

Revised January 20, 2016

Accepted January 21, 2016

\section{Correspondence}

Jin-Soo Kang

Kangskin Dermatology Clinic, 1673-2 Seocho-

dong, Seocho-gu, Seoul 06334, Korea

Tel: +82-2-584-9007

Fax: +82-2-521-4384

E-mail: kangskinahanmail.net

(C) Korean Society for Laser Medicine and Surgery

(c) This is an open access article distributed under the terms of the Creative Commons Attribution NonCommercial License (http://creativecommons.org/ licenses/by-nc/4.0) which permits unrestricted noncommercial use, distribution, and reproduction in any medium, provided the original work is properly cited.
Continuous movements with muscle contraction and UV induced photodamage causes periorbital wrinkles. The periorbital area is a sensitive and anatomically vulnerable lesion, making treatment difficult. Various treatment options have been used to improve periorbital wrinkles but the results are variable and there is no single optimal treatment. Demands for minimally invasive treatment with reduced downtime and fewer side effects are growing, and radiofrequency devices have been effectively introduced for the treatment of periorbital wrinkles. In the current report, we treated three patients with variable degrees of periorbital wrinkles using an invasive non-insulated microneedle pulsed electric signal device and met remarkable improvements with a very short downtime without crust formation, bruising, or other side effects.

\section{Key words}

Periorbital wrinkles; Electric signal 


\section{INTRODUCTION}

Muscle contraction and UV-induced photoaging results in periorbital wrinkles, which are often firstly noticed as an aging sign. As the orbicularis oculi muscle contracts by various facial expressions and movements, folding or wrinkling of the overlying skin occurs intensifying the UVinduced photodamage. ${ }^{1,2}$ Changes of tissue volume by an aging process can additionally accentuate the morphological changes of the orbital area. The periorbital area is a particular lesion to treat due to its unique characteristics that it has continuous movements and that the eyelid skin is the thinnest part among body skin with about 0.04 $\mathrm{mm}$ thickness of its epidermis, and also because of its important function. ' According to the anatomical vulnerability, treatment of periorbital wrinkles should be performed cautiously to avoid any adverse events including functional damage. ${ }^{1}$ Treatments including botulinum toxin injection, filler injection, dermabrasion, chemical peeling, various laser therapies, and applying topical agents such as tretinoin have been used to treat periorbital wrinkles, but the results are variable and there is no single optimal treatment. ${ }^{3,4}$

Radiofrequency (RF) devices can deliver thermal energy into the dermis which induces collagen regeneration and contraction, resulting in dermal rejuvenation and skin tightening. ${ }^{5}$ Various RF systems have been safely used to treat periorbital wrinkles in the literature and was verified with its effectiveness for periorbital rejuvenation. 2,3,6 It has recently been shown that not only non-invasive RF systems but also invasive RF systems even with noninsulated electrodes can also safely deliver RF energy to the skin while preserving the epidermis. ${ }^{7.8}$ In this report, we present three patients with different grades of periorbital wrinkles which were improved after treatment with a novel invasive pulsed electric signal device with noninsulated microneedle electrodes.

\section{CASE REPORT}

Three patients with periorbital wrinkles were treated with an invasive bipolar pulsed electric signal RF device (Sylfirm; Viol, Kyunggi, Korea), which uses disposable tips composed of 25 minimally invasive non-insulated microneedle electrodes uniformly arranged in a $5 \times 5$ pattern. ${ }^{8}$ Each patient presented with variable degrees of fine wrinkles on the corner of the eyes, lower and upper eyelids. The first patient was a 66-year-old Korean female presenting with many deep lines nearly covering the entire periorbital area, with coarse wrinkles extending into the cheek. She also showed fat loss on the periorbital area causing a sunken eye appearance (Fig. 1). The second patient was a 49-year-old Korean female and presented with shallow wrinkles with some deeper wrinkles
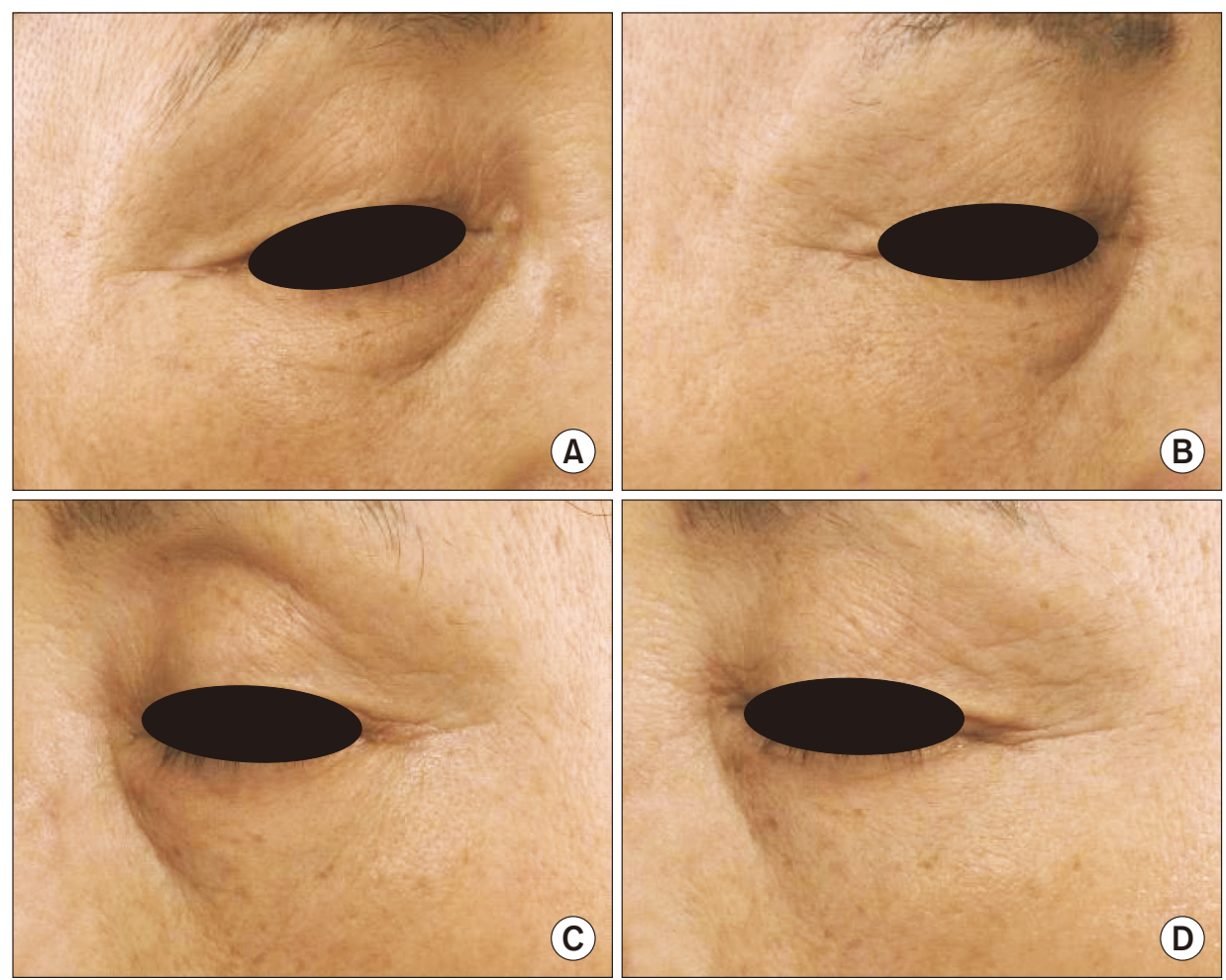

Fig. 1. Clinical photographs showing many deep wrinkles nearly covering the entire periorbital area, with coarse wrinkles extending into the cheek of a 66-year-old Korean female, right and left periorbital area (A), (C) before and (B), (D) two weeks after two sessions of bipolar invasive RF treatments respectively. 
on the corners with skin thinning (Fig. 2). The last patient was a 45-year-old Korean female who was concerned about some shallow wrinkles with a deeper line starting from the right medial canthus extending downwards on her right lower eyelid (Fig. 3). All patients did not have any pertinent family history or medical history including fat,
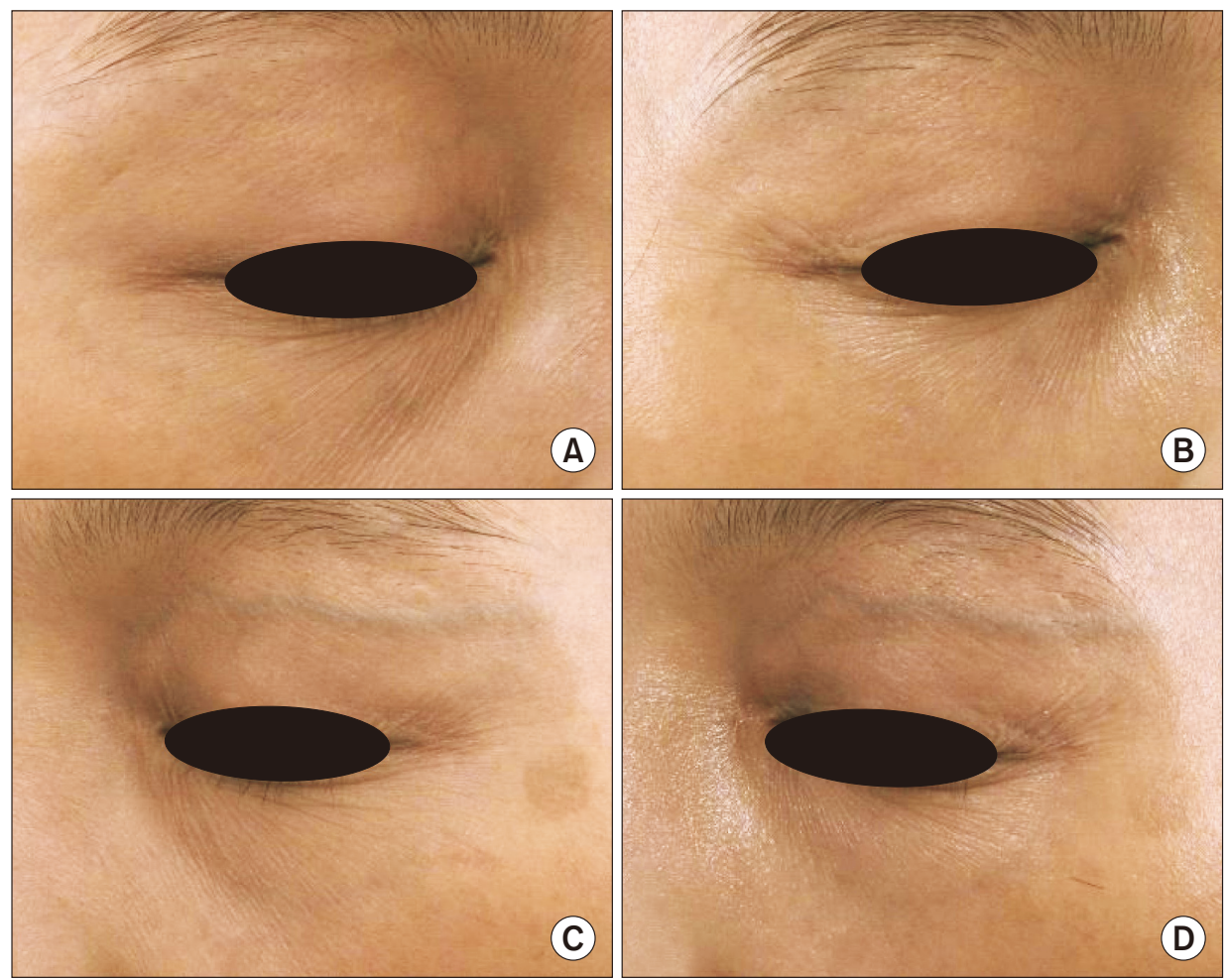

D)
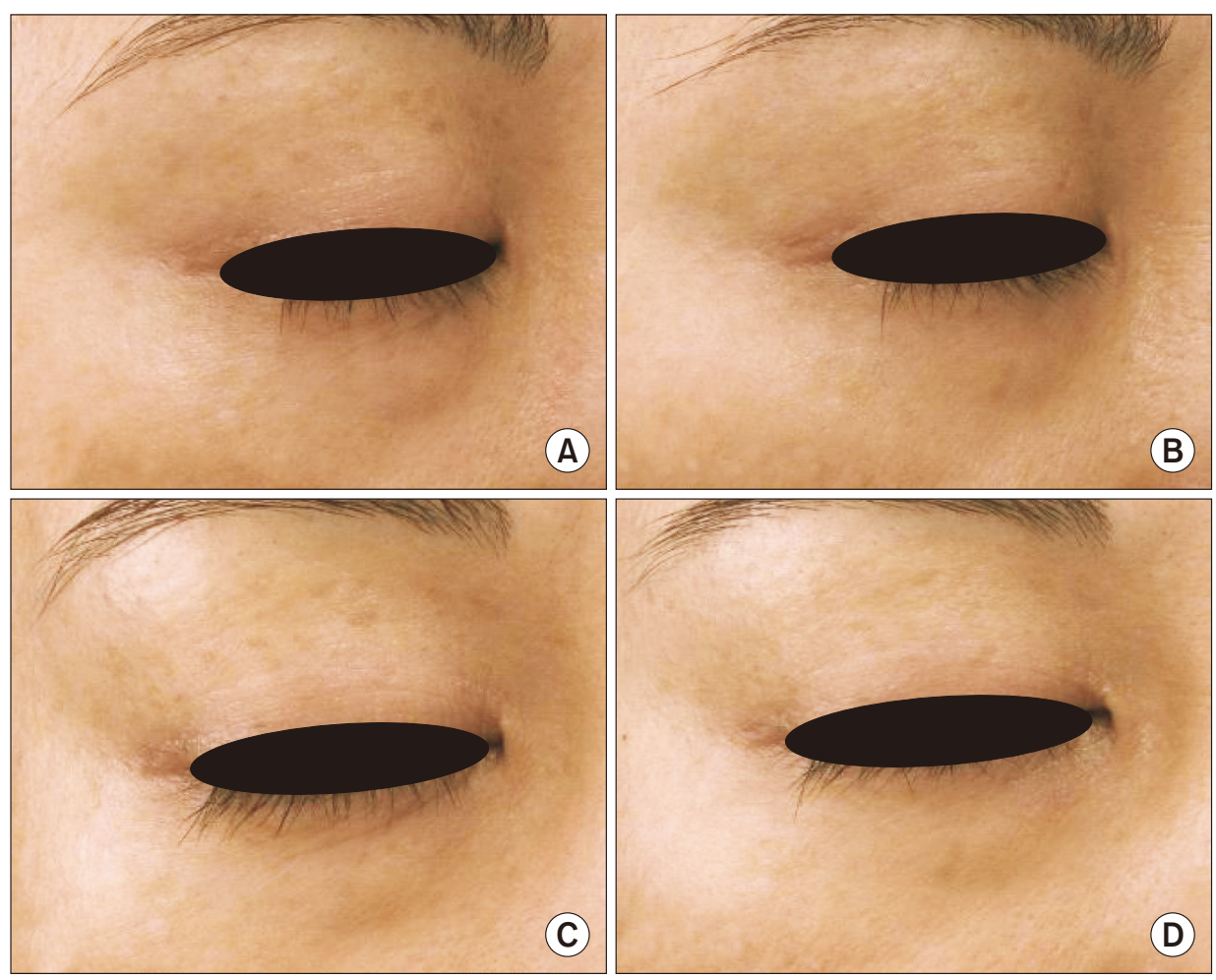

Fig. 2. Clinical photographs showing shallow wrinkles with some deeper wrinkles on the corner of the eyes of a 49-year-old Korean female, right and left periorbital area (A), (C) before and (B), (D) two weeks after two sessions of bipolar invasive RF treatments respectively.

Fig. 3. Clinical photographs showing shallow wrinkles with a deeper line starting from the right medial canthus extending downwards on her right lower eyelid of a 45-yearold Korean female, (A), (C) before and (B), (D) two weeks after two sessions of bipolar invasive RF treatments. 
collagen or filler injection and prior laser treatment to treat periorbital wrinkles.

After obtaining a written informed consent, the face was cleansed and a topical anesthetic cream with a eutectic mixture of 2.5\% lidocaine and 2.5\% prilocaine (EMLA; AstraZeneca Canada Inc., Ontario, Canadal was applied on the periorbital area under occlusion for 30 minutes. After removing EMLA, the invasive bipolar RF treatment was performed with a power level of 3-4, penetration depth of $1.0-1.5 \mathrm{~mm}$ for a single pass with $<10 \%$ overlap. Then, a restoration serum containing human fibroblast conditioned media (O MEDIA; Scarlet RF Inc., San Juan Capistrano, CA, USA) was applied on the periorbital area. Immediately after the treatment, slight swelling and erythema was seen on the treated lesions which resolved within a few hours. No noticeable side effects including epidermal shedding, crust formation or bruising was observed, but patients reported mild to moderate pain during the procedure. Two treatment sessions were performed with a 1 month interval, and digital photographs were taken at baseline and at 2 weeks after the last treatment. All patients showed objective improvements with their periorbital wrinkles with only 2 sessions and all accompanied patient's subjective satisfaction.

\section{DISCUSSION}

Among various treatment modalities which can be used to improve photoaged skin, ablative resurfacing including dermabrasion or laser treatments can be very effective, but demands towards treatments with reduced or minimal downtime and less adverse events have been growing. ${ }^{6}$ This is also true when treating the periorbital area because this lesion particularly is a sensitive area with anatomical vulnerability and it greatly affects facial appearance. Therefore, options of less invasive treatment are becoming more popular including minimally invasive RF treatments for periorbital wrinkle treatment.

Invasive RF system penetrates the skin by microneedles and directly delivers thermal energy into the dermis inducing collagen contraction and coagulation. Microneedling itself also causes physical stimulation, and the treatment induces a wound healing process in overall which induces dermal collagen regeneration and remodeling resulting in skin tightening and improvement of skin laxity and wrinkles. ${ }^{5}$ As the microneedles are very thin and as the thermal coagulation can be limited to the tip of the electrode, dermal remodeling and rejuvenation can be gained more effectively avoiding unnecessary epidermal damage compared to noninvasive RF devices. ${ }^{7-10}$ Effective
RF energy delivery into the dermis by generating coagulation columns separately around each microneedle while preserving the epidermis has also been demonstrated by the invasive bipolar non-insulated microneedle RF device. ${ }^{8}$ Microneedling can also induce elastin deposition together with neocollagenesis reversing solar elastosis caused by photodamage. ${ }^{9-11}$

There are previous studies reporting experiences of microneedle RF devices in the treatment of periorbital wrinkles, but these studies used insulated microneedle RF devices. ${ }^{2,36}$ As far as we know, this is the first report using a bipolar non-insulated microneedle RF device for the treatment of periorbital wrinkles. In this report, we presented three patients with periorbital wrinkles which were treated with a novel invasive non-insulated pulsed electric signal device. Acceptable improvements of periorbital wrinkles were observed in a short period with only two sessions of treatment without crust, bruising or any other side effects. No patients reported postinflammatory hyperpigmentation after treatment. The mechanism by which this RF device improved periorbital wrinkles in such a short period is not investigated in this study. Although further studies using objective grading scales and measurements also including histological studies are required to accurately analyze the mechanism and treatment effects, this invasive bipolar non-insulated microneedle RF device can be a good option for treating periortibal wrinkles with minimal downtime and a relatively lower risk of side effects and patient discomfort.

\section{REFERENCES}

1. Manaloto RM, Alster TS. Periorbital rejuvenation: a review of dermatologic treatments. Dermatol Surg 1999;25:1-9.

2. Jeon IK, Chang SE, Park GH, Roh MR. Comparison of microneedle fractional radiofrequency therapy with intradermal botulinum toxin a injection for periorbital rejuvenation. Dermatology 2013;227:367-72.

3. Lee SJ, Kim JI, Yang YJ, Nam JH, Kim WS. Treatment of periorbital wrinkles with a novel fractional radiofrequency microneedle system in dark-skinned patients. Dermatol Surg 2015;41:615-22.

4. Fu JJ, Hillebrand GG, Raleigh P, Li J, Marmor MJ, Bertucci V, et al. A randomized, controlled comparative study of the wrinkle reduction benefits of a cosmetic niacinamide/peptide/retinyl propionate product regimen vs. a prescription $0.02 \%$ tretinoin product regimen. Br J Dermatol 2010;162:647-54.

5. Elsaie ML, Choudhary S, Leiva A, Nouri K. Nonablative radiofrequency for skin rejuvenation. Dermatol Surg 2010;36: 577-89. 
6. Kim JK, Roh MR, Park GH, Kim YJ, Jeon IK, Chang SE. Fractionated microneedle radiofrequency for the treatment of periorbital wrinkles. J Dermatol 2013;40:172-6.

7. Taheri A, Mansoori P, Sandoval LF, Feldman SR, Williford PM, Pearce D. Entrance and propagation pattern of high-frequency electrical currents in biological tissues as applied to fractional skin rejuvenation using penetrating electrodes. Skin Res Technol 2014;20:270-3.

8. Na J, Zheng Z, Dannaker C, Lee SE, Kang JS, Cho SB. Electromagnetic initiation and propagation of bipolar radiofrequency tissue reactions via invasive non-insulated microneedle electrodes. Sci Rep 2015;5:16735.

9. Choi M, Choi S, Kang JS, Cho SB. Successful treatment of refractory melasma using invasive micro-pulsed electric signal device. Med Lasers 2015;4:39-44.

10. Hantash BM, Ubeid AA, Chang H, Kafi R, Renton B. Bipolar fractional radiofrequency treatment induces neoelastogenesis and neocollagenesis. Lasers Surg Med 2009;41:1-9.

11. Aust MC, Fernandes D, Kolokythas P, Kaplan HM, Vogt PM. Percutaneous collagen induction therapy: an alternative treatment for scars, wrinkles, and skin laxity. Plast Reconstr Surg 2008;121:1421-9. 